\title{
Factores asociados a la violencia conyugal hacia la mujer en el Perú, utilizando Regresión Logística
}

\section{Wilman Josue Límaco Ninahuanca ${ }^{1}$ y Olga Lidia Solano Dávila ${ }^{2}$}

Resumen: La presente investigación tuvo por objetivo identificar y analizar los factores asociados a la violencia contra la mujer en el Perú, utilizando el modelo de regresión logística. Se utilizó un enfoque cuantitativo, no experimental, transversal y explicativo tomando como base la Encuesta Demográfica y Salud Familiar del año 2013 del Instituto Nacional de Estadística e Informática, y cuya muestra probabilística incluyó a 22920 mujeres en edad fértil de 15 a 49 años. Los resultados obtenidos a nivel descriptivo evidenciaron que la violencia contra la mujer en el Perú ocurre principalmente a través de la violencia psicológica - verbal $(67,5 \%)$, lo cual representó casi el doble de la violencia física $(35,7 \%)$ y más de ocho veces la violencia sexual $(8,4 \%)$. También, se construyó un modelo de regresión logística, y aunque los mismos presentaron limitaciones en la especificad y sensibilidad, se comprobó que variables como consumo de alcohol del esposo, que ella actualmente trabaje y problema si quedo embarazada, fueron factores homogéneos y concurrentes en los tres modelos desarrollados para violencia psicológica - verbal, física y sexual.

Palabras clave: Modelo de regresión logística; violencia contra la mujer; ENDES.

\section{Factors associated with conjugal violence in the emergency center woman}

\begin{abstract}
The purpose of the present investigation was to identify and analyse the factors associated with violence against women in Peru, using logistic regression model. A quantitative, non-experimental, transversal and explanatory approach was used, based on the Demographic and Family Health Survey of the year 2013 of the National Institute of Statistics and Informatics, and whose probabilistic sample included 22920 women of childbearing age from 15 to 49 years. The results obtained at the descriptive level showed that violence against women in Peru occurs mainly through psychological - verbal violence $(67,5 \%)$, which represented almost twice the physical violence $(35,7 \%)$ and more than eight times sexual violence $(8,4 \%)$. Also, a logistic regression model was built, and although they presented limitations in the specificity and sensitivity, i was verified that variables such as husband's alcohol consumption, that she currently works and problem if I am pregnant, were homogeneous and concurrent factors in the three models developed for psychological violence- verbal, physical and sexual.
\end{abstract}

Keywords: Logistic regression model; violence against; ENDES.

Recibido: 06/09/2019. Aceptado: 16/12/2019. Publicado online: 26/12/2019.

(CLos autores. Este artículo es publicado por la Revista PESQUIMAT de la Facultad de Ciencias Matemáticas, Universidad Nacional Mayor de San Marcos. Este es un artículo de acceso abierto, distribuido bajo los términos de la licencia Creative Commons Atribucion-No Comercia-Compartir Igual 4.0 Internacional.(http://creativecommons.org/licenses/by-nc-sa/4.0/) que permite el uso no comercial, distribución y reproducción en cualquier medio, siempre que la obra original sea debidamente citada. Para información, por favor póngase en contacto con revistapesquimat.matematica@unmsm.edu.pe

\footnotetext{
${ }^{1}$ MTC, Ministerio de Transportes y Comunicaciones, e-mail: wlimaco@mtc.gob.pe

${ }^{2}$ UNMSM, Facultad de Ciencias Matemáticas, e-mail: osolanod@unmsm.edu.pe
} 


\section{Introducción}

En la actualidad, la violencia contra la mujer se ha convertido en un tema de debate público, los medios de comunicación informan cada día acerca de nuevos episodios de agresiones, maltratos y amenazas en contra la autonomía de la mujer, hay una transgresión hacia sus derechos, así como un contexto de desigualdad y menosprecio donde convergen diversos factores de índole social, cultural, económico y demográfico. Las estadísticas ilustran dicha situación, donde a nivel global se ha estimado que el $35 \%$ de las mujeres en el mundo han sido víctimas de violencia física y/o sexual [9], y esta cifra se eleva en el Perú, hasta alrededor del $70 \%$ para violencias del tipo psicológica, física y sexual [8].

A raíz de tales hechos y con el objeto de luchar contra la violencia de género, fue que en el año 1993 la Organización de las Naciones Unidas (ONU) aprobó la Declaración sobre la eliminación de la violencia contra la mujer, y luego en el año 1999 declaró que cada 25 de noviembre se conmemore el Día Internacional de la Eliminación de la Violencia contra la Mujer. A nivel Perú, también se implementaron políticas públicas para la protección de la mujer con la promulgación de la Ley $\mathrm{N}^{o} 28983$ sobre la igualdad de oportunidades entre mujeres y varones (15 de marzo del 2007), la Ley $\mathrm{N}^{\circ} 30364$ para prevenir, sancionar y erradicar la violencia contra las mujeres y los integrantes del grupo familiar (el 22 de noviembre del 2015), la creación del Programa Nacional Contra la Violencia Familiar y Sexual, la creación de Centros de Emergencia Mujer (CEM), la Línea 100, Chat 100, entre otras acciones ejecutadas tanto a nivel de gobierno como también por parte de las instituciones privadas y sin fines de lucro.

Basado en lo antes mencionado y teniendo presente la repercusión negativa que siguen soportando las mujeres, sea desde una perspectiva de salud, laboral o educativo, es que se ha considerado necesario seguir investigando el ciclo reiterativo de violencia de género que aún sigue persistiendo muy a pesar de los esfuerzos que se vienen impulsando, siendo así el objetivo de la presente investigación identificar y analizar los factores asociados a la violencia contra la mujer en las modalidades física, psicológica y sexual.

\section{Metodología}

\section{Regresión Logística Múltiple}

Según Hosmer, Lemeshow, y Sturdivant (2013) en los métodos de regresión "muy a menudo, la variable de resultado es discreta, tomando dos o más valores posibles. El modelo de regresión logística es el modelo de regresión más utilizado para el análisis de estos datos".

En ese sentido, sea $Y$ una variable dependiente dicotómica con valores 0 y 1 y $X$ un vector de $p$ variables independientes denotado por $X=\left(X_{1}, X_{2}, \cdots, X_{p}\right)$. También, sea $\pi(x)=E(Y / x)$ la media condicional de $Y$ dada $x$ cuando se usa la distribución logística.

El modelo de regresión logística se expresa de la siguiente forma:

$$
\pi(x)=\frac{e^{\beta_{0}+\beta_{1} x_{1}+\beta_{2} X_{2}+\cdots+\beta_{p} X p}}{1+e^{\beta_{0}+\beta_{1} x_{1}+\beta_{2} X_{2}+\cdots+\beta_{p} X p}} .
$$

De la ecuación (1) se requiere aplicar una transformación que permita expresar el modelo como una relación lineal, y en ese sentido, la transformación logit linealiza dicha expresión que consiste en dividir la proporción de éxitos, $\pi(x)$, entre uno menos la proporción de éxitos $(1-\pi(x))$ y luego aplicarle el logaritmo a este cociente.

$$
\ln \left(\frac{\pi(x)}{1-\pi(x)}\right)=\beta_{0}+\beta_{1} X_{1}+\beta_{2} X_{2}+\cdots+\beta_{p} X p
$$




\section{Estimación de los parámetros}

Se utiliza el método de máxima verosimilitud para la estimación de los parámetros del modelo de regresión logística, que consiste en maximizar $l(\beta)$, donde el vector $\beta=\left(\beta_{0}, \beta_{1}, \beta_{2}, \cdots, \beta_{p}\right)$.

Se considera una muestra de $n$ observaciones independientes $(X i, Y i), i=1,2, \ldots, n$. Dado que $X=\left(X_{1}, X_{2}, \cdots, X_{p}\right)$ son independientes, la función de verosimilitud se puede escribir de la siguiente forma:

$$
l(\beta)=\prod_{i=1}^{n} \pi\left(x_{i}\right)^{y_{i}}\left[1-\pi\left(x_{i}\right)\right]^{1-y_{i}} .
$$

A la ecuación (3), se aplica el logaritmo a la función de verosimilitud.

$$
\ln [l(\beta)]=\sum_{i=1}^{n}\left\{y_{i} \ln \left[\pi\left(x_{i}\right)\right]+\left(1-y_{i}\right) \ln \left[1-\pi\left(x_{i}\right)\right]\right\}
$$

De la ecuación (4), la expresión se deriva e iguala a cero para encontrar los valores de $\beta=\left(\beta_{0}, \beta_{1}, \beta_{2}, \ldots, \beta_{p}\right)$,

$$
\begin{gathered}
\sum_{i=1}^{n}\left[y_{i}-\pi\left(x_{i}\right)\right]=0 \\
\sum_{i=1}^{n} x_{i j}\left[y_{i}-\pi\left(x_{i}\right)\right]=0
\end{gathered}
$$

La solución de las ecuaciones (5) y (6) se resuelven mediante métodos iterativos que disponen los softwares estadísticos.

\section{Evaluación del modelo}

La evaluación del modelo se basa en la estadística G, también llamada desvianza, que estudia la importancia de las variables independientes en el modelo.

Sean las hipótesis:

$$
\begin{aligned}
& H_{0}: \quad \beta_{0}=\beta_{1}=\beta_{2}=\cdots=\beta_{p}=0 \\
& H_{1}: \quad \text { Al menos una } \beta_{i} \text { es diferente de cero. }
\end{aligned}
$$

La estadística $\mathrm{G}$ se define de la siguiente forma:

$$
\begin{aligned}
G & =\text { Desvianza(modelo sin variables) }- \text { Desvianza(modelo con variables) } \\
& =-2 \ln \left[\frac{\text { verosimilitud del modelo sin variables }}{\text { verosimilitud del modelo con variables }}\right] \\
& =2\left\{\sum_{i=1}^{n}\left[y_{i} \ln \left(\pi_{i}\right)+\left(1-y_{i}\right) \ln \left(1-\pi_{i}\right)\right]-\left[n_{1} \ln \left(n_{1}\right)+n_{0} \ln \left(n_{0}\right)-n \ln (n)\right]\right\},
\end{aligned}
$$

donde

$$
n_{1}=\sum_{i=1}^{n} y_{i} \text { y } n_{0}=\sum_{i=1}^{n}\left(1-y_{i}\right)
$$

La estadística G tiene una distribución Chi-Cuadrado con $p$ grados de libertad, bajo la hipótesis nula. Rechazamos $H_{0}$ a un nivel de significancia $\alpha$, si:

$$
G>\chi_{(p)}^{2}
$$




\section{Estadística de Wald}

La estadística Wald evalúa la significancia de cada uno de los parámetros del modelo. Se considera las siguientes hipótesis:

$$
\begin{array}{ll}
H_{0}: & \beta i=0 \\
H_{1}: & \beta i \neq 0 .
\end{array}
$$

La estadística de Wald se define como:

$$
W=\widehat{\beta}^{\prime}\left[\widehat{\operatorname{Var}}(\widehat{\beta})_{T}\right]^{-1} \widehat{\beta}
$$

De la ecuación (7), la estadística de Wald se distribuye como una variable aleatoria Chi Cuadrado con $p$ grados de libertad, bajo la hipótesis nula. Rechazamos $H_{0}$ a un nivel de significancia $\alpha$, si:

$$
W>\chi_{(p)}^{2}
$$

También, si el p-valor, es menor que 0,05, se rechaza la hipótesis nula y se concluye que el parámetro es diferente de cero y por tanto es significativo.

\section{Odds Ratio}

Odds Ratio $(O R)$, también llamado razón de momios $(\mathrm{RM})$ o razón de ventajas. De la ecuación (2), se aplica el antilogaritmo para obtener:

$$
\left(\frac{\pi(x)}{1-\pi(x)}\right)=e^{\beta_{0}+\beta_{1} X_{1}+\beta_{2} X_{2}+\ldots+\beta_{p} X p}
$$

Luego, el $O R$ que indica la relación entre el factor $X_{i}$ y la variable respuesta $Y$ es dado por:

$$
O R_{i}=e^{\beta_{i}}
$$

donde los intervalos de confianza están dados por

$$
\exp \left[\beta_{i} \pm Z_{1-\alpha / 2} * \widehat{S E}(\beta i)\right]
$$

Se interpreta como:

- Si $O R=1$, implica que no hay relación entre el factor $X_{i}$ y la variable respuesta $Y$.

- Si $O R>1$, el factor $X_{i}$ es un factor de riesgo, es decir, hay más posibilidad o chance de un resultado Y dado que el factor (de riesgo) está presente.

- Si $O R<1$, el factor $X_{i}$ es un factor de protección, es decir, hay menos posibilidad o chance de un resultado $Y$ dado que el factor (de protección) está presente.

\section{Tipo y diseño de investigación}

El tipo de investigación fue bajo un enfoque cuantitativo, con un diseño no experimental, transversal y correlacional - causal.

\section{Unidad de análisis}

La unidad de análisis fue una mujer en edad fértil de 15 a 49 años de edad, ubicadas lo largo del territorio nacional. 


\section{Recolección de datos}

Se utilizó la información consignada en la Encuesta Demográfica y de Salud Familiar del año 2013 del Instituto Nacional de Estadística e Informática, cuya muestra fue equivalente a 22,920 mujeres para el capítulo Violencia Familiar.

\section{Variables}

De acuerdo con los datos recopilados en la ENDES 2013, las variables respuestas estudiadas fueron Violencia Psicológica-Verbal contra la Mujer, Violencia Física contra la Mujer y Violencia Sexual contra la Mujer. Dichas variables fueron contrastadas con las variables explicativas demográficas (Estado civil, Región, Tipo de lugar de residencia, Edad de la mujer), sociales (Problema si quedó embarazada, Esposo vive en casa, Esposo bebe alcohol, Frecuencia esposo bebe alcohol, Nivel de educación del esposo) y económicos (Índice de riqueza, actualmente ella trabaja)

\section{Resultados y Discusión}

\subsection{Análisis descriptivo}

La violencia contra la mujer en el Perú se dio principalmente bajo una perspectiva psicológica y/o verbal $(67,5 \%)$, seguido por la violencia física $(35,7 \%)$ y sexual $(8,4 \%)$, y teniendo una reducción poco significativa en los últimos cuatro años.

Tabla 1: Violencia contra la mujer en el Perú, según tipo, año $2010-2013$

\begin{tabular}{|l|c|c|c|c|}
\hline \multicolumn{1}{|c|}{ Violencia } & 2010 & 2011 & 2012 & 2013 \\
\hline Psicológica y/o verbal & $72,1 \%$ & $70,0 \%$ & $70,6 \%$ & $67,5 \%$ \\
\hline Física & $37,7 \%$ & $38,0 \%$ & $36,4 \%$ & $35,7 \%$ \\
\hline Sexual & $8,6 \%$ & $9,3 \%$ & $8,7 \%$ & $8,4 \%$ \\
\hline
\end{tabular}

$\mathrm{Al}$ analizar por tipo de violencia, tenemos que aquellas mujeres con estado civil divorciada, separada o viuda $(86,8 \%)$ y que pertenecen al segundo quintil de riqueza $(72,5 \%)$ son susceptibles de violencia psicológica y/o verbal. Para el caso de la violencia física, esto se da básicamente en mujeres de 40 a 44 años (40,9\%), divorciadas, separadas o viudas $(56,7 \%)$, con educación secundaria $(39,5 \%)$ y que pertenecen al segundo quintil de riqueza $(42,9 \%)$. A nivel de la violencia sexual, ésta usualmente se presenta en mujeres de 40 a 44 años de edad (10,8\%), divorciadas, separadas o viudas $(23,0 \%)$, sin educación $(10,4 \%)$ y que pertenecen al segundo quintil de riqueza $(11,7 \%)$. (ver Tabla 2$)$. 
Tabla 2: Violencia contra la mujer en el Perú, según características, año 2013

\begin{tabular}{|c|c|c|c|}
\hline \multirow[t]{2}{*}{ Características } & Tipos de & Violencia & (Porcentaje) \\
\hline & Psicológica y/o verbal & Física & Sexual \\
\hline Total & 67,5 & 35,7 & 8,4 \\
\hline \multicolumn{4}{|l|}{ Grupo de edad } \\
\hline $15-19$ & 68,5 & 20,5 & 5,8 \\
\hline $20-24$ & 64,8 & 28,5 & 5,4 \\
\hline $25-29$ & 66,7 & 32,5 & 6,4 \\
\hline $30-34$ & 68,3 & 37,0 & 8,3 \\
\hline $35-39$ & 67,1 & 36,2 & 9,1 \\
\hline $40-44$ & 68,3 & 40,9 & 10,8 \\
\hline $45-49$ & 68,7 & 39,6 & 10,2 \\
\hline \multicolumn{4}{|l|}{ Estado conyugal } \\
\hline Casada/Conviviente & 64,1 & 32,1 & 5,9 \\
\hline Divorciada/Separada/Viuda & 86,8 & 56,7 & 23,0 \\
\hline \multicolumn{4}{|l|}{ Nivel de educación } \\
\hline Sin educación & 63,1 & 35,9 & 10,4 \\
\hline Primaria & 68,7 & 37,3 & 9,4 \\
\hline Secundaria & 69,1 & 39,5 & $\overline{9,2}$ \\
\hline Superior & 64,1 & 27,8 & 5,9 \\
\hline \multicolumn{4}{|l|}{ Quintil de riqueza } \\
\hline Quintil inferior & 67,0 & 31,9 & 8,2 \\
\hline Segundo quintil & 72,5 & 42,9 & 11,7 \\
\hline Quintil intermedio & 68,9 & 37,9 & 8,9 \\
\hline Cuarto quintil & 66,9 & 37,9 & 7,3 \\
\hline Quintil superior & 59,5 & 24,2 & 4,9 \\
\hline
\end{tabular}

Nota: Quintil de riqueza: Bienestar de cada hogar que depende de la disponibilidad de bienes, servicios, y vivienda.

\subsection{Comprobación de hipótesis}

Se ajustaron tres modelos de regresión logística con relación a la violencia contra la mujer en sus modalidades psicológica-verbal, física y sexual, y se observó que variables como frecuencia esposo bebe alcohol, actualmente ella trabaja y problema si quedó embarazada, fueron factores homogéneos y concurrentes en los tres modelos desarrollados que explicaron significativamente los tipos de violencia antes mencionados.

\section{Psicológica - Verbal contra la mujer}

Según la Tabla 3, el modelo de regresión logística para la violencia psicológica - verbal resultó ser significativo $\left(G=7,367>X_{(1 ; 0,95)}^{2}=3,841 ; p=0,000<0 ; 05\right.$, lo cual nos ha permitido deducir que al menos una de las variables explicativas propuestas en el modelo ha contribuido a explicar la presencia de la violencia psicológica-verbal contra la mujer. En conformidad con lo antes mencionado, la estadística de Wald ha permitido ratificar la importancia de cada una de las cinco variables explicativas propuestas: Estado civil, Actualmente ella trabaja, Problema si quedó embarazada, Frecuencia esposo bebe alcohol, Índice de riqueza 
Tabla 3: Variables en la ecuación en el modelo de regresión logística para la Violencia PsicológicaVerbal contra la mujer, año 2013

\begin{tabular}{|c|c|c|c|c|c|c|}
\hline \multirow[t]{2}{*}{ Variable } & \multirow[t]{2}{*}{$\beta$} & \multirow[t]{2}{*}{ Wald } & \multirow[t]{2}{*}{ p-valor } & \multirow[t]{2}{*}{$\operatorname{Exp}(\beta)$} & \multicolumn{2}{|l|}{ I.C.95\% } \\
\hline & & & & & L. Inferior & L.Superior \\
\hline \multicolumn{7}{|l|}{ Estado civil } \\
\hline Conviviente & 0,217 & 12,661 & 0,000 & 1,242 & 1,102 & 1,400 \\
\hline \multicolumn{7}{|l|}{ Actualmente ella trabaja } \\
\hline Sí & 0,161 & 7,394 & 0,007 & 1,174 & 1,046 & 1,319 \\
\hline \multicolumn{7}{|l|}{ Problema si quedó embarazada } \\
\hline $\begin{array}{c}\text { No pueda quedar embarazada/ } \\
\text { No tiene sexo }\end{array}$ & 0,088 & 0,025 & 0,876 & 1,092 & 0,364 & 3,277 \\
\hline Gran problema & 0,268 & 17,508 & 0,000 & 1,307 & 1,153 & 1,482 \\
\hline Pequeño problema & 0,229 & 7,680 & 0,006 & 1,257 & 1,069 & 1,477 \\
\hline \multicolumn{7}{|l|}{ Frecuencia esposo bebe alcohol } \\
\hline Algunas veces & 0,562 & 51,442 & 0,000 & 1,754 & 1,504 & 2,045 \\
\hline Frecuentemente & 1,963 & 104,725 & 0,000 & 7,120 & 4,889 & 10,369 \\
\hline \multicolumn{7}{|l|}{ Índice de riqueza } \\
\hline Muy pobre & 0,316 & 8,057 & 0,005 & 1,372 & 1,103 & 1,707 \\
\hline Pobre & 0,400 & 13,068 & 0,000 & 1,492 & 1,201 & 1,854 \\
\hline Medio & 0,391 & 11,481 & 0,001 & 1,478 & 1,179 & 1,852 \\
\hline Rico & 0,259 & 4,650 & 0,031 & 1,296 & 1,024 & 1,640 \\
\hline Constante & $-0,441$ & 12,691 & 0,000 & 0,644 & & \\
\hline
\end{tabular}

Nota: Categoría de referencia: Estado civil (Casada), Actualmente ella trabaja (No), Problema si quedó embarazada (No hay problema),Frecuencia esposo bebe alcohol (Nunca), índice de riqueza (Muy rico). Estadística $G=7,367, g l=1, X^{2}=3,841, p=0,000$.

Wald: Si p-valor es menor que 0,05 , el coeficiente asociado a la variable es significativo. Clasificación del modelo: Global $=69,5 \%$, Sensibilidad $=98,3 \%$, Especificidad $=3,9 \%$.

También precisar que, aunque la capacidad de clasificación del modelo presentó limitaciones ( sensibilidad $=98,3 \%$, especificidad $=3,9 \%$ ), se identificaron ORś relevantes que manifestaron como principal razón de violencia psicológica-verbal contra la mujer la frecuencia del consumo de alcohol por parte del esposo, y que en términos estadísticos, se interpreta de la siguiente forma: Aquellas mujeres con esposo que frecuentemente beben alcohol tienen 7,1 (IC (95\%): 4,889-10,369) más posibilidades de violencia psicológica-verbal frente a las mujeres con esposo que nunca beben alcohol.

Otras razones de violencia psicológica-verbal contra la mujer ocurren debido al estado civil, donde las mujeres que son convivientes tienen 1,242(IC (95\%): 1,102- 1,400) más posibilidades de violencia psicológica-verbal frente a las mujeres que son casadas. Situación similar se tuvo en el aspecto laboral, donde las mujeres que trabajan tienen 1,174 (IC (95\%): 1,046-1,319) más posibilidades de violencia psicológica-verbal frente a las mujeres que no trabajan.

Respecto a la fertilidad, las mujeres que consideran un gran problema quedar embarazada tienen 1,307 (IC (95\%): 1,153- 1,482) más posibilidades de violencia psicológica-verbal frente a las mujeres que consideran que no hay problema. Similarmente, para las mujeres que lo consideran un pequeño problema, la chance de violencia psicológica-verbal fue de 1,257 (IC (95\%): 1,069 - 1,477), y con respecto a las mujeres que no pueden quedar embarazadas o no tienen sexo, la chance de violencia psicógica - verbal se situó en 1,092 (IC (95\%): ,364- 3,277). 
Finalmente, en referencia al índice de riqueza, las mujeres económicamente pobres tienen 1,372 (IC (95\%): 1,103-1,707) más chance de violencia psicológica-verbal con respecto a las mujeres de mejor condición económica (muy ricas). Situación similar se observó con las mujeres muy pobres, medios, y ricos, llegando incluso a representar casi doble riesgo de violencia psicológica-verbal si tomamos como referencia el intervalo superior de confianza.

Dado esto, la ecuación de regresión logística para la Violencia Psicológica - Verbal contra la mujer se formula de la siguiente manera:

$$
P(\text { Violencia Psicológica }- \text { Verbal }=S i)=\frac{1}{1+e^{-Z}},
$$

donde:

$Z=-0,441+0,217 *$ Conviviente $+0,161 *$ Ellasitrabaja $+0,088 *$ No puede quedar embarazada $+0,268 *$ Gran problema quedar embarazada $+0,229 *$ Pequeño problema quedar embarazada $+0,562 *$ Esposo algunas veces bebe alcohol $+1,963 *$ Esposo frecuentemente bebe alcohol $+0,316 *$ Muy probre $+0,400 *$ Pobre $+0,391 *$ Medio $+0,259 *$ Rico

\section{Violencia Física contra la mujer}

Según como se detalla en la Tabla 4, se desarrolló un modelo significativo de regresión logística para la violencia física contra la mujer $\left(G=33,631>X_{(6 ; 0,95)}^{2}=12,5916 ; p=0,000<0,05\right)$ y se identificaron cinco variables explicativas: Actualmente ella trabaja, Problema si quedó embarazada, Frecuencia esposo bebe alcohol, Grupos quinquenales e Índice de riqueza.

Precisar que la capacidad de clasificación del modelo también presentó limitaciones (sensibilidad $=23,7 \%$, especificidad $=92,4 \%$ ), sin embargo, se obtuvieron ORs significativos mediante los cuales se logró identificar que el principal factor determinante de violencia física contra la mujer fue el consumo de alcohol por parte del esposo, donde aquellas mujeres con esposo que frecuentemente beben alcohol tienen 10,526 (IC (95\%): 7,775-14,251) más posibilidades de violencia física frente a las mujeres con esposo que nunca beben alcohol.

También se identificaron otros factores y entre ellos se observó que aquellas mujeres con una condición económica pobre tienen 2,224 (IC(95\%): 1,757 - 2,815) más posibilidades de violencia física frente a mujeres con una condición económica muy rica, y de igual manera ocurre con la edad (grupos quinquenales) dado que aquellas mujeres con edades de 40-44 años tienen 2,023 (IC (95\%): 1,431- 2,859) más posibilidad de violencia física frente a mujeres con edades de 15-19 años 
Tabla 4: Variables en la ecuación del modelo de regresión logística para la Violencia Física contra la mujer, año 2013

\begin{tabular}{|c|c|c|c|c|c|c|}
\hline \multirow[t]{2}{*}{ Variable } & \multirow[t]{2}{*}{$\beta$} & \multirow[t]{2}{*}{ Wald } & \multirow[t]{2}{*}{ p-valor } & \multirow[t]{2}{*}{$\operatorname{Exp}(\beta)$} & \multicolumn{2}{|c|}{ I.C.95\% $\operatorname{Exp}(\beta)$} \\
\hline & & & & & Inferior & Superior \\
\hline \multicolumn{7}{|l|}{ Actualmente ella trabaja } \\
\hline $\mathrm{Si}$ & 0,334 & 31,097 & 0,000 & 1,396 & 1,242 & 1,570 \\
\hline \multicolumn{7}{|l|}{ Problemas si quedó embarazada } \\
\hline $\begin{array}{c}\text { No puede quedar embarazada/ } \\
\text { No tiene sexo }\end{array}$ & 0,042 & 0,005 & 0,942 & 1,043 & 0,337 & 3,228 \\
\hline Gran problema & 0,480 & 56,327 & 0,000 & 1,616 & 1,425 & 1,831 \\
\hline Pequeño problema & 0,323 & 15,980 & 0,000 & 1,382 & 1,179 & 1,619 \\
\hline \multicolumn{7}{|l|}{ Frecuencia esposo bebe alcohol } \\
\hline Algunas veces & 0,478 & 29,906 & 0,000 & 1,613 & 1,359 & 1,914 \\
\hline Frecuentemente & 2,354 & 231,934 & 0,000 & 10,526 & 7,775 & 14,251 \\
\hline \multicolumn{7}{|l|}{ Grupos quinquenales } \\
\hline $20-24$ años & 0,238 & 1,804 & 0,179 & 1,296 & 0,896 & 1,796 \\
\hline 25-29 años & 0,454 & 7,093 & 0,008 & 1,574 & 1,127 & 2,198 \\
\hline 30-34 años & 0,566 & 11,143 & 0,001 & 1,762 & 1,263 & 2,457 \\
\hline 35-39 años & 0,527 & 9,324 & 0,002 & 1,694 & 1,208 & 2,377 \\
\hline 40-44 años & 0,705 & 15,928 & 0,000 & 2,023 & 1,431 & 2,859 \\
\hline 45-49 año & 0,686 & 13,943 & 0,000 & 1,986 & 1,385 & 2,847 \\
\hline \multicolumn{7}{|l|}{ Índice de riqueza } \\
\hline Muy pobre & 0,356 & 8,508 & 0,004 & 1,427 & 1,124 & 1,813 \\
\hline Pobre & 0,799 & 44,175 & 0,000 & 2,224 & 1,757 & 2,815 \\
\hline Medio & 0,604 & 23,390 & 0,000 & 1,829 & 1,432 & 2,337 \\
\hline Rico & 0,469 & 12,806 & 0,000 & 1,598 & 1,236 & 2,066 \\
\hline Constante & $-2,543$ & 146,668 & 0,000 & 0,079 & & \\
\hline
\end{tabular}

Nota: Categoría de referencia: Actualmente ella trabaja (No), Problema si quedó embarazada (No hay problema), Frecuencia esposo bebe alcohol (Nunca), Grupos quinquenales (15-19 años), índice de riqueza (Muy rico). Estadística $\mathrm{G}=33,631, \mathrm{gl}=6, X^{2}=1,5916, p=0,000$.

Wald: Si p-valor es menor que 0,05 , el coeficiente asociado a la variable es significativo. Clasificación del modelo: Global $=66,0 \%$, Sensibilidad $=23,7 \%$, Especificidad $=92,4 \%$.

Dado esto, la ecuación de regresión logística para la Violencia Física contra la mujer se formula de la siguiente manera:

$$
P(\text { Violencia Física }=S i)=\frac{1}{1+e^{-Z}}
$$

donde:

$Z=-2,543+0,334 *$ Ella si trabaja $+0,042 *$ No puede quedar embarazada $+0,480 *$ Gran problema quedar embarazada $+0,323 *$ Pequeño problema quedar embarazada $+0,478 *$ Esposo algunas veces bebe alcohol $+2,354 *$ Esposo frecuentemente bebe alcohol $+0,686 * \operatorname{Edad}[45-49]$ $+0,238 * \operatorname{Edad}[20-24]+0,454 * \operatorname{Edad}[25-29]+0,566 * \operatorname{Edad}[30-34]+0,527 * \operatorname{Edad}[35-39]$ $+0,705 * \operatorname{Edad}[40-44]+0,356 *$ Muy pobre $+0,799 *$ Pobre $+0,604 *$ Medio $+0,469 *$ Rico 


\section{Violencia Sexual contra la mujer}

Tabla 5: Variables en la ecuación del modelo de regresión logística para la Violencia Sexual contra la mujer, año 2013

\begin{tabular}{|c|c|c|c|c|c|c|}
\hline \multirow[t]{2}{*}{ Variable } & \multirow[t]{2}{*}{$\beta$} & \multirow[t]{2}{*}{ Wald } & \multirow[t]{2}{*}{ p-valor } & \multirow[t]{2}{*}{$\operatorname{Exp}(\beta)$} & \multicolumn{2}{|c|}{ I.C.95\% $\operatorname{Exp}(\beta)$} \\
\hline & & & & & Inferior & Superior \\
\hline \multicolumn{7}{|l|}{ Actualmente ella trabaja } \\
\hline $\mathrm{Si}$ & 0,334 & 7,940 & 0,005 & 1,397 & 1,107 & 1,762 \\
\hline \multicolumn{7}{|l|}{ Problemas si quedó embarazada } \\
\hline $\begin{array}{l}\text { No puede quedar embarazada/ } \\
\text { No tiene sexo }\end{array}$ & 0,297 & 0,076 & 0,783 & 1,346 & 0,162 & 11,174 \\
\hline Gran problema & 0,685 & 27,251 & 0,000 & 1,984 & 1,534 & 2,565 \\
\hline Pequeño problema & 0,506 & 9,348 & 0,002 & 1,658 & 1,199 & 2,293 \\
\hline \multicolumn{7}{|l|}{ Frecuencia esposo bebe alcohol } \\
\hline Algunas veces & 0,531 & 6,628 & 0,010 & 1,701 & 1,135 & 2,548 \\
\hline Frecuentemente & 2,073 & 80,614 & 0,000 & 7,952 & 5,057 & 12,504 \\
\hline \multicolumn{7}{|l|}{ Grupos quinquenales } \\
\hline $20-24$ años & 0,004 & 0,000 & 0,992 & 1,004 & 0,450 & 2,240 \\
\hline 25-29 años & 0,415 & 1,160 & 0,281 & 1,514 & 0,712 & 3,218 \\
\hline 30-34 años & 0,503 & 1,725 & 0,189 & 1,653 & 0,781 & 3,500 \\
\hline 35-39 años & 0,645 & 2,815 & 0,093 & 1,906 & 0,897 & 4,048 \\
\hline 40-44 años & 0,869 & 5,063 & 0,024 & 2,385 & 1,119 & 5,085 \\
\hline 45-49 año & 0,866 & 4,820 & 0,028 & 2,378 & 1,097 & 5,153 \\
\hline \multicolumn{7}{|l|}{ Índice de riqueza } \\
\hline Muy pobre & 0,838 & 8,189 & 0,004 & 2,312 & 1,302 & 4,103 \\
\hline Pobre & 1,040 & 12,788 & 0,000 & 2,830 & 1,600 & 5,004 \\
\hline Medio & 0,996 & 11,188 & 0,001 & 2,706 & 1,510 & 4,850 \\
\hline Rico & 0,373 & 1,330 & 0,249 & 1,452 & 0,770 & 2,736 \\
\hline Constante & $-5,329$ & 113,921 & 0,000 & 0,005 & & \\
\hline
\end{tabular}

Nota: Categoría de referencia: Actualmente ella trabaja (No), Problema si quedó embarazada (No hay problema), Frecuencia esposo bebe alcohol (Nunca), Grupos quinquenales (15-19 años), índice de riqueza (Muy rico). Estadística $G=23,917, g l=6, X^{2}=12,5916, p=0,001$.

Wald: Si p-valor es menor que 0,05, el coeficiente asociado a la variable es significativo.Clasificación del modelo: Global $=93 \%$, Sensibilidad $=100 \%$, Especificidad $=0 \%$

Según la Tabla 5, se comprobó la significancia estadística del modelo de regresión logística para la violencia sexual contra la mujer $\left(G=23,917>X_{(6 ; 0,95)}^{2}=12,5916 ; p=0,000<0 ; 05\right)$, y se identificaron cinco variables explicativas como determinantes del modelo: Actualmente ella trabaja, Problema si quedó embarazada, Frecuencia esposo bebe alcohol, Grupos quinquenales e Índice de riqueza.

Aunque, la capacidad de clasificación del modelo también presentó limitaciones ( sensibilidad $=100,0 \%$, especificidad $=0,00 \%$ ), los OR's presentados nos permitieron identificar a la variable "Frecuencia esposo bebe alcohol" como factor determinante asociado a la violencia sexual contra la mujer, y cuya interpretación estadística fue la siguiente: Aquellas mujeres con esposo que frecuentemente beben alcohol tienen 7,952 (IC (95\%): 5,057- 12,504) más posibilidades de episodios de violencia sexual en comparación con aquellas mujeres con esposo que nunca beben alcohol. 
Otro factor asociado a la violencia sexual se da con aquellas mujeres que consideran un gran problema quedar embarazada, presentando un riesgo de1,984 (IC (95\%): 1,534- 2,565) frente a las mujeres que no consideran un problema el embarazo. También, si tomamos como referencia a las mujeres en edad de 40-44 años, el riesgo de violencia sexual para ellas es de 2,385 (IC (95\%): 1,119-5,085) mayor frente a las mujeres en edad de 15-19 años, y con respecto a la condición económica, se identificó que las mujeres en condiciones económica pobre tiene 2,830 IC (95\%): 1,600 - 5,004) más posibilidades de violencia sexual en comparación con las mujeres de mejor condición económica (muy ricas).

Dado esto, la ecuación de regresión logística para la Violencia Sexual es el siguiente:

$$
P(\text { Violencia Sexual }=S i)=\frac{1}{1+e^{-Z}},
$$

donde:

$Z=-5,329+0,334 *$ Ella si trabaja $+0,297 *$ No puede quedar embarazada $+0,685 *$ Gran problema quedar embarazada $+0,506 *$ Pequeño problema quedar embarazada $+0,531 *$ Esposo algunas veces bebe alcohol $+2,073 *$ Esposo frecuentemente bebe alcohol $+0,866 * \operatorname{Edad}[45-49]$ $+0,004 * \operatorname{Edad}[20-24]+0,415 * \operatorname{Edad}[25-29]+0,503 * \operatorname{Edad}[30-34]+0,645 * \operatorname{Edad}[35-39]$ $+0,869 * \operatorname{Edad}[40-44]+0,838 *$ Muy pobre $+1,040 *$ Pobre $+0,996 *$ Medio $+0,373 *$ Rico

\section{Conclusión}

Los resultados obtenidos permiten concluir que el principal tipo de violencia contra la mujer en el Perú fue la violencia psicológica -verbal (67.5\%), seguido por la violencia física (35.7\%) y la violencia sexual $(8.4 \%)$. También se evidenció a través de la construcción de tres (03) modelos de regresión logística (violencia psicológica - verbal, física y sexual), que la violencia contra la mujer se debe básicamente a la presencia de las variables "Frecuencia esposo bebe alcohol", "Actualmente ella trabaja" y "Problema si quedo embarazada".

\section{Referencias bibliográficas}

[1] CEPAL (2002). Violencia contra la mujer en relación de pareja: América Latina y el Caribe: Una propuesta para medir su magnitud y evolución. Santiago de Chile, Chile: Naciones Unidas.

[2] Gervilla E. , Jiménez, R., Montaño, J. J., Sesé, A., Cajal, B. y Palmer, A. (2008). The Methodology of Data Mining. An Application to alcohol consumption in teenagers. Revista Adicciones, 21, (1), 65-80. Recuperado de http://adicciones.es/index.php/adicciones/article/viewFile/253/242.

[3] Hosmer, D.W., Lemeshow, S., \& Sturdivant, R.X.(2013). Applied logistic Regression. New Jersey: Jhon Wiley \& Sons

[4] Instituto Nacional de Estadística e Informática (2009). Factores asociados a la presencia de violencia contra la mujer. Lima, Perú: Talleres de la Oficina Técnica de Administración del INEI.

[5] Instituto Nacional de Estadística e Informática (2009). Modelos Multivariados para la violencia conyugal, sus consecuencias y la solicitud de ayuda. Lima, Perú: Talleres de la Oficina Técnica de Administración del INEI 
[6] Ministerio de la Mujer y Desarrollo Social (2010). Plan Nacional contra la violencia hacia la mujer, 2009-15. Lima, Perú: Industrias Gráficas Ausangate S.A.C..

[7] Montgomery, D., Peck, L. y Vining, G.(2006). Introducción al análisis de regresión lineal. (3era ed.). San Juan Tlihuaca, México: Compañía Editorial Continental

[8] Observatorio Nacional de la Violencia contra las Mujeres y los integrantes del grupo familiar (2018). Indicadores de la Encuesta Demográfica y de Salud Familiar (ENDES). Recuperado de https://observatorioviolencia.pe/datos-inei-2017-2/

[9] Organización Mundial de la Salud (2013). Estimaciones mundiales y regionales de la violencia contra la mujer, Prevalencia y efectos de la violencia conyugal y de la violencia sexual no conyugal en la salud. Recuperado de https://apps.who.int/iris/bitstream/handle/10665/85239/9789241564625_eng.pdf?sequence=1

[10] Tristán, F. (2005), La Violencia contra la mujer: Feminicidio en el Perú. Lima, Perú: Ymagino Publicidad S.A.C. 